\title{
FLUORESCENT LINE EMISSION OF MOLECULAR HYDROGEN
}

John H. Black

Steward Observatory, Tucson, AZ 85721

Ewine F. van Dishoeck

Center for Astrophysics, Cambridge, MA 02138

The electric quadrupole vibration-rotation transitions of molecular hydrogen can be excited either by thermal collisions in regions of high temperature or by ultraviolet absorption and fluorescence that leaves the molecules in excited levels, v>0, of the electronic ground state in interstellar clouds located very close to hot stars. Predictions of this fluorescent emission (Gould and Harwit 1963, Black and Dalgarno 1976) have only recently been confirmed by observations in Orion (Hayashi et al. 1985) and toward the reflection nebula NGC 2023 (Gatley and Kaifu 1987). Most previous observations of the $2 \mu \mathrm{m} \mathrm{H}_{2}$ lines have been consistent with thermal excitation in shocked regions?

We summarize here some results of theoretical calculations of the infrared spectrum of $\mathrm{H}_{2}$ excited by ultraviolet fluorescence. Detailed models of interstellar clouds (van Dishoeck and Black 1986) are used to describe the abundance and excitation of $\mathrm{H}_{2}$ as functions of depth through the boundary layers of a cloud exposed to intense ultraviolet starlight. Because the rates of ultraviolet absorption decrease rapidly with increasing optical depth in the electronic transitions, both the abundance and excitation of $\mathrm{H}_{2}$ are severely depth dependent. The fluorescent line intensities are proportional to the excited-state concentrations integrated over path length through a cloud. These intensities are most sensitive to the flux of ultraviolet radiation at the boundary and depend to a lesser extent on density and temperature within the cloud. At moderate resolution $(\lambda \Delta \lambda \simeq 100)$ in the $2 \mu \mathrm{m}$ wavelength region, the fluorescent emission is distinguishable from collisionally excited thermal emission, primarily through the high relative intensity of the $\left(v^{\prime}, v^{\prime \prime}\right)=(2,1)$ and $(3,2)$ lines, as shown in Figure 1. The distinction is even more pronounced in the 1.0-1.8 $\mathrm{mm}$ region, where fluorescent features of comparable intensity appear but thermal features at relevant temperatures are virtually absent.

In the future, it may be possible to detect the fluorescent $\mathrm{H}_{2}$ line emission from unresolved ensembles of young, hot stars and molecular clouds in other galaxies. The $\mathrm{H}_{2}$ line emission can be used as a measure of star forming activity because the fluorescent line flux depends upon the mean ultraviolet intensity (i.e. the number of hot, massive stars) and upon the total surface area in molecular clouds. 


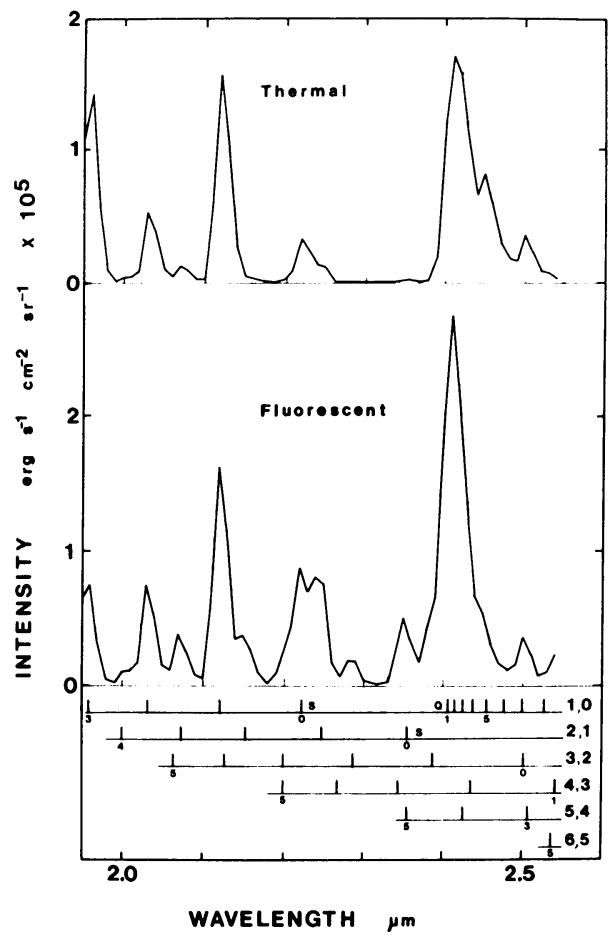

Figure 1 shows theoretical $H_{2}$ emission line spectra that have been convolved with a Gaussian having FWHM $=0.02 \mu \mathrm{m}$. The fluorescence spectrum has been computed for a uniform cloud of dens ity $3000 \mathrm{~cm}^{-3}$ and temperature $30 \mathrm{~K}$ with an ultraviolet flux of $4.5 \times 10^{-5}$ photons $\mathrm{cm}^{-2} \mathrm{~s}^{-1} \mathrm{~Hz}$ at $1000 \AA$, which is 1000 times the mean interstellar radiation field. These parameters ware chosen to reproduce the observations of NGC 2023 (Gatley and Kaifu 198/). and the radiation field corresponds to illumination by the B1.5 V star, HD 37903, at a distance of $0.6 \mathrm{pC}$. A thermal spectrum computed for $\mathrm{T}=2000 \mathrm{~K}$ is shown for comparison: the column density of $\mathrm{H}_{2}$ in this case was chosen to produce the same intensity in the $(1,0) S(1)$ line at $2.122 \mu \mathrm{m}$ as in the fluorescent spectrum. The positions of S- and Q-branch lines are indicated at the bottom.

\section{REFERENCES}

Black, J. H. and Dalgarno, A. 1976, Ap. J., 203, 132.

Gatley, I. and Kaifu, N. 1987, in Astrochemistry, IAU Symposium

No. 120, M. S. Vardya and S. P. Tarafdar, eds., to be published. Gould, R. J. and Harwit, M. 1963, Ap. J., 137, 694.

Hayashi, M., Hasegawa, T., Gatley, I., Garden, R., and Kaifu, N. 1985, M. N. R. A. S., 215,31 . van Dishoeck, E. F. and Black, J. H. 1986, Ap. J. Suppl., in press. 
HASEGAWA: What is the range of the intensity ratio between the $v=2-1$ and $v=1-0 \mathrm{~S}(1)$ lines in your model? Is it possible to tell whether $\mathrm{H}_{2}$ is excited by a shock or by UV radiation based on that ratio?

VAN DISHOEK: The ratio is 0.57 in the fluorescent model of the reflection nebula NGC 2023. This value is quite insensitive to the conditions in the cloud. If the $\mathrm{H}_{2}$ molecules were excited by collision in a shock at a temperature of $2000 \mathrm{~K}$, the ratio would be much lower, about 0.1 .

INDUCED STAR FORMATION IN M17: HIGH RESOLUTION $\mathrm{NH}_{3}$ AND IR OBSERVATIONS

\author{
M. Felli, M. Massi \\ Osservatorio di Arcetri, Florence, Italy \\ R. Stanga \\ Instituto di Astronomia, Florence, Italy \\ E. Churchwe11 \\ University of Wisconsin, Madison, USA
}

A VLA radio continuum study of the HII region M17 (Felli, Churchwell and Massi, 1984) has shown the presence of an elongated sharp arc structure in the South Bar of the nebula, in a region of heavy obscuration. The arc has been interpreted as an ionization boundary, viewed edge on, located between the diffuse H II region, to the east, and a dense component of the extended molecular cloud, to the west. About $3^{\prime \prime}$ to the west of this arc, an ultra-compact H II region has been found. This has a shell type structure, a linear diameter of $0.004 \mathrm{pc}$ and probably is the result of induced star formation in the molecular cloud produced by the pressure front preceding the ionization front.

In this paper, we present preliminary results of VLA observations of the $(1,1) \mathrm{NH}_{3}$ line emission from the molecular component facing the ionization front and $3.6 \mathrm{~m}$ ESO mapping of the same region at 10 and 17.4 $\mu \mathrm{m}$, aimed to resolve the emission of the ultra-compact component from that of the ionization front.

The $\mathrm{NH}_{3}$ emission (spatial resolution 5", velocity resolution 1.2 $\mathrm{km} \mathrm{s}^{-1}$ ) comes primarily from a 60" $\times 30^{\prime \prime}$ region about $30^{\prime \prime}$ to the west of the arc structure and is bounded on the east side by the ionization front. Isolated $\mathrm{NH}_{3}$ blobs are also present to the east of the arc. The $\mathrm{NH}_{3}$ emission is highly clumped and shows distinct velocity components. In particular, near the peak of emission there are two velocity components separated by $2 \mathrm{~km} \mathrm{~s}^{-1}$ and spatially adjacent. The main line optical

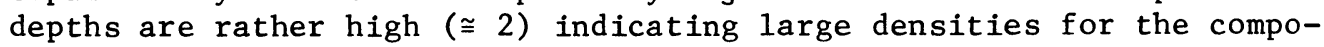
nents. These molecular blobs represent sub-condensations in the molecular cloud which will, eventually, be triggered to collapse by the shock front preceding the ionization front. No $\mathrm{NH}_{3}$ feature is directly associated to the ultra-compact HII region, or to the IR sources. 